\title{
Multiple Parenchymal Neurocysticercosis: A Case Report
}

\author{
KC I', Rana KJ1', Joshi R¹, Mandal A², Bhhatarai S² \\ ${ }^{1}$ Consultant Physician, Shree Birendra Hospital, ${ }^{2}$ Resident, Shree Birendra Hospital
}

\begin{abstract}
Cysticercosis is a parasitic infection with CNS involvement in $60-90 \%$ of infested patients. The larval form of pork intestinal tapeworm (Taenia solium) is responsible for cysticercosis. Humans are the definitive hosts and usually harbor the adult tapeworm in small intestine as an asymptomatic infestation. Neurocysticercosis most commonly affects the brain parenchyma. Solitary and multiple cysts in brain parencyma is common but we came across a 24 year old lady patient with huge number of cysts which is relatively rare. The aim of this article is to report the rare case.
\end{abstract}

Key words: Cysticercosis, Neurocysticercosis, Taenia solium

\section{Case profile}

A 24 yrs teetotaler housewife from Nepalgunj was reffered to our hospital with progressive holocranial headache associated with episodic vomiting and repeated loss of consciousness. The patient was in her usual state of health $1 \frac{1}{2}$ yrs back when she began to develop gradual onset dull headache limiting her daily activities with the severity scale of 4 out of 10 . Headache remained throughout the day followed by nausea and vomiting, 2-3 episodes a day. She started having loss of consciousness 10 weeks back associated with limb stiffness, up rolling of eyeball and frothing from mouth followed by severe headache, amnesia, generalized weakness and increased sleep. No history of fever, cough, chest pain, shortness of breath, palpitation, loss of appetite or weight loss.

Clinically vitals were normal, no papilloedema or signs of raised intracranial pressure and systemic examination was normal. CBC, ESR, RBS, LFT'S, HIV-I,II Urine Re, ECG, CXR were normal. However Toxoplasma antibody was positive, titer suggestive of past infection. Stool examination showed Giardia lamblia cyst but no ova of tapeworm. CT and MRI Head showed multiple scattered opacities in the brain parenchyma suggestive of NCC in various stages, no hydrocephalus.

She was started on Albendazole $15 \mathrm{mg} / \mathrm{kg} /$ day for 14 days along with prednisolone $1 \mathrm{mg} / \mathrm{kg} /$ day $X 7$ days then gradually tapered over next few days along with Valproic-acid 300mg twicw daily, Amitryptaline 10mg at bed time, Antiemetic and analgesics. Vomiting and headache persisted till 15th post admission days however seizure was controlled. Appetite and sleep became normal and headache disappeared after day 15 and she was discharged on day 16th with advise to continue anticonvulsant with follow up after 2 months with repeat MRI. In subsequent follow up patient was improving and has not reported seizure and headache.

\section{Discussion}

Neurocysticercosis (NCC) is the common parasitic infection worldwide. Active NCC is classified according to the location into: Parenchymal cysts, Ventricular cysts, Subarachnoid cysts and Cysticercus racemose ${ }^{1}$.

Clinical presentation of NCC is quite variable and depends on: (i) location, growth, size and number of cysts; (ii) the stage of cyst - vesicular, degenerative, calcific; and (iii) host immune response ${ }^{2}$. Seizures are the most common presentation of NCC. Diagnosis of NCC is mostly based on the clinical, radiological, immunological and epidemiological parameters ${ }^{3}$. The

\section{Address for Correspondence:}

Name: Dr. Indu KC

E-mail:drindukc@hotmail.com

Ph. №.: 9851013800 

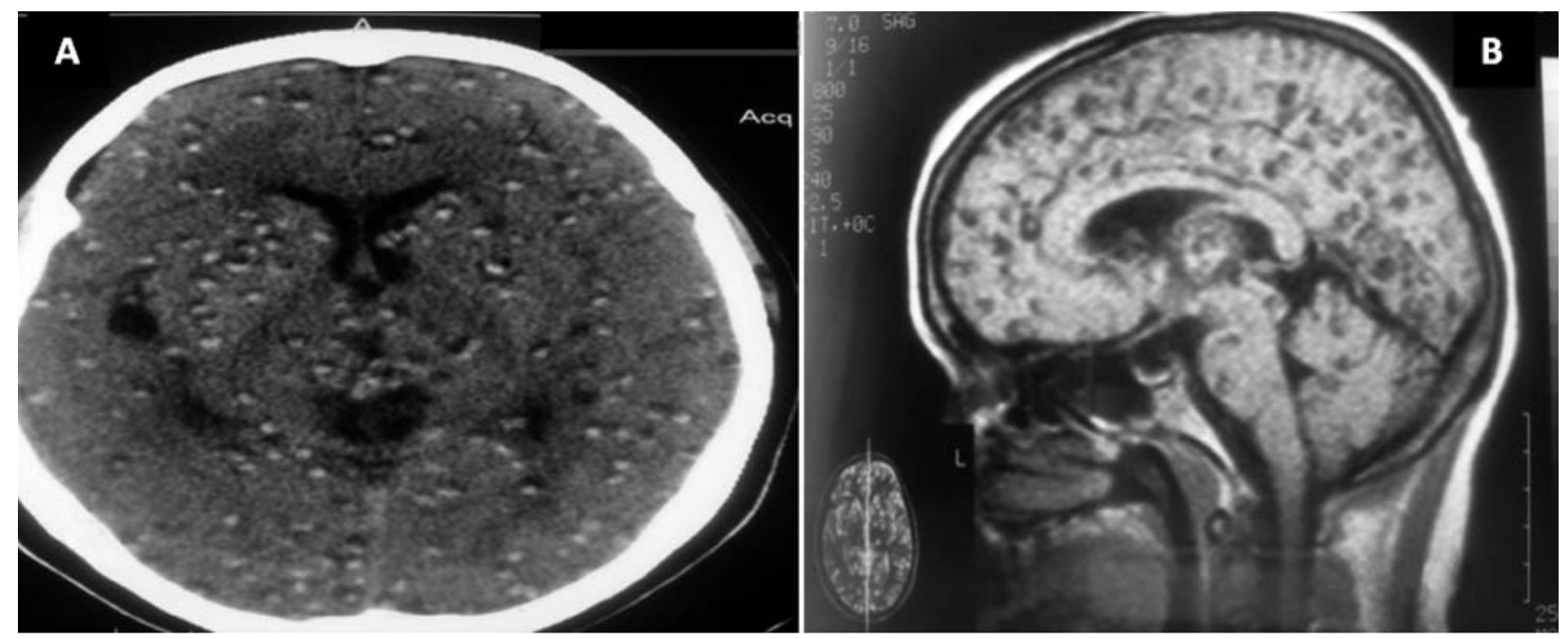

Fig 1: A. CT Head showing multiple neurocysticercosis, B. MRI showing multiple Hypodence opacities all over the parenchyma in different stages of larval growth

diagnostic serological test of choice in NCC is electroimmuno transfer blot assay (EITB). It has near 100\% specificity and $94-98 \%$ sensitivity in patients with two or more cysts ${ }^{4}$. MRI is the radiological investigation of choice to determine the pathological stage of $\mathrm{cyst}^{2}$. The stage of the cyst determines the MRI cyst morphology: Viable cysts show neither enhancement nor perilesional edema, and are hypointense to brain parenchyma. Contrast enhancement and perilesional edema are the MRI characteristics of cysts in the degenerative phase. The larval cyst is recognizable as a cystic structure but is obviously irreversibly damaged, and cystic fluid becomes more turbid, corresponding to the colloidal vesicular stage described by Escobar. In our case the diagnosis was not difficult as the patient has very charecteristic presenting clinical features and her CT and MRI was almost diagnostic. The un common feature of our case was the multiplicity of the lesion and its spread to all the parenchyma of brain. Very few such widespread lesions have been reported in literature and for the first time we came across with such a huge number of cysts in brain parenchyma.

Control of seizure with anticonvulsant becomes easy after treatment with antihelmentic however there is controversy whether to use or not use the antihelmentic drugs in the treatment of neurocystecercosis ${ }^{5}$. Antiepileptic therapy can be stopped once the follow up CT shows resolution of the lesion but if seizure occurs after resolution of oedema and resorption or calcification of the degenerating cyst, such patient require long term antiepileptic therapy.Important differential diagnosis is CNS Toxoplasmosis which is treated with combination of Sulfadiazine, Pyrimethamine and folic acid. Clindamycin is substituted for those who does not tolerate Sulfadizine.

\section{Conclusion}

Neurocystecercosis is a treatable condition provided it is diagnosed well in time and most patients recover fully after treatment. Treatment is medical and surgery is indicated in patient with mass effect and raised intracranial pessure.

\section{References}

1. Martinez HR, Range GR, Arrendondo-Estrada JH, Marfil A, Onofre J. Medical and surgical treatment in neurocysticercosis: A MR study in 161 cases. J Neurol Sci 1995May;130(1):25-34.

2. Nash TE. Human case management and treatment of cysticercosis. Acta Trop 2003;87:61-9.

3. Del Brutto $\mathrm{OH}$, Rajshekhar V, White AC Jr, Tsang VC, Nash TE, Takayanagui OM, et al. Proposed diagnostic criteria for neurocysticercosis. Neurology 2001;57:177-83.

4. Garg RK. Diagnostic criteria for neurocysticercosis; some modifications are needed for Indian patients. Neurol India 2004;52:171-7.

5. Karen IR, Kenneth LT. Nonbacterial CNS infection, Harrison's Principles of Internal Medicine2006(II),2471-2487.

6. Osborne AG. Diagnostic neuroradiology, ed. USA: Mosby yearbook, Inc. 1994: 709-710. 